\title{
DEFICIENCY OF THE MESENTERY OVER THE LOWER ILEUM.
}

\author{
By GEORGE E. ARMSTRONG, Montrear.
}

By one of those strange coincidences that we are all familiar with, the two patients presenting the rare anomaly that I now report were in the Royal Victoria Hospital, Montreal, at the same time. The anomaly consists in the absence of a mesentery for the terminal six or seven inches of the ileum (Figs. 280, 281).

My own case was a man, age 33 years, sent up to me as a case of recurring appendicitis. He gave a history of having had three attacks at intervals of a month or six weeks. The outstanding feature in his history was the severity of the pain during the attacks. He told us that during the last illness, from which he was just recovering, morphia failed to give relief, and that he had been kept under the influence of ether from 11 p.m. until 6 a.m.

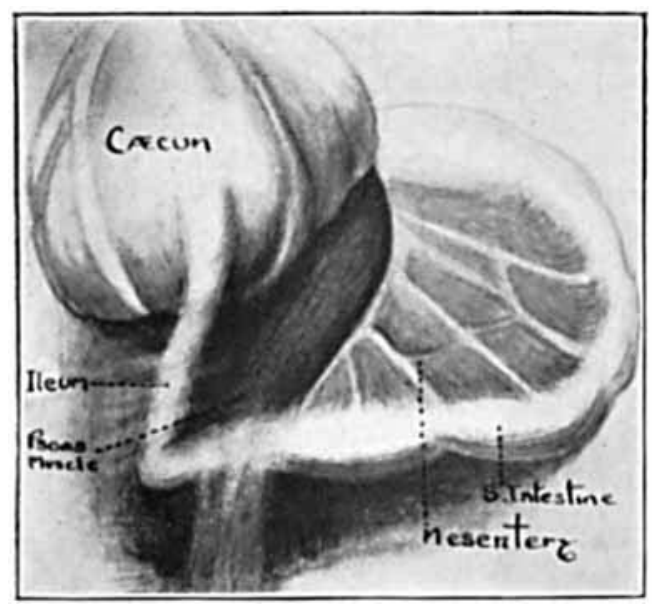
the following day. He referred all his trouble to the region of the appendix, and on admission the right lower quadrant was definitely tender. Seventy-two hours after

FIGs. - 80,281 . - Showing ahence of mesentery at terminal part of ileun.

admission I proceeded to uncover the region of the appendix. The condition observed on opening the abdomen was most unusual. The cæcum was very mobile, and on pushing it and the ascending colon inwards, the tissues outside the colon were found to be hæmorrhagic, echymosed, and at one spot the peritoneum was found lacerated. The appendix was curled up external to the ascending colon, and retroperitoneal. It was congested. The area external to the colon looked almost exactly as if he had been kicked with a heavy boot. The terminal six inches of the ileum was subperitoneal. The deficiency in the mesentery began in the pelvis in front of the sacro-iliac synchondrosis, about an inch below the brim. From this point the ileum passed up over the external iliac vein and over the psoas muscle to its junction with the cæcum. It was very intimately adherent to the wall of the external iliac vein by means of normal tissue. There was no sign of inflammatory action. The appendix was removed, and cacum and ascending colon were sutured to the outer abdominal wall by three rows of sutures. So far there has not been any recurrence of symptoms.

The other case was discovered at autopsy. The patient had been under the care of 
one of my colleagues for a condition not associated with the anomaly. On tracing the small gut downwards, it was found to be free from adhesions or exudate, and apparently

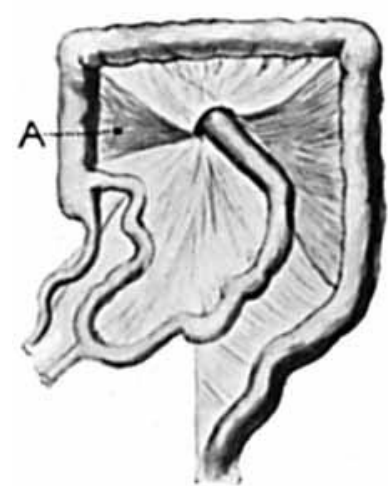

114:. 282.-Normal mesentery. (A) Normal extent of fusion of mesocolon to posterior ahdominal wall. ended suddenly at the brim of the pelvis over the right sacro-iliac joint. On lifting up the cæcum, which was markedly dilated, it was found to be very mobile, with a definite mesocolon, and to be apparently disconnected from the ileum. This was due to an anomalous condition of the last six inches of ileum. The mesentery ended abruptly at the brim of the pelvis over the right sacro-iliac joint, where the ileum became retroperitoneal and ran posteriorly across the iliopsoas muscle, then turned upwards for nearly three inches, and lay in the groove between the iliopsoas and the quadratus lumborum. It then reached the posterior surface of the cæcum, on which it ran downwards for about two inches to approximately its normal insertion at the ileocxcal valve. In this manner, with the cæcum in its normal position, it ran a course forming a loop with rather a sharp turn or kink at its point of junction with the posterior aspect of the cæcum. This whole loop, when the crcum was first lifted up, was completely flattened, and so not noticeable; thus causing the appearance of the abrupt ending of the ileum at the pelvic brim. Manipulation of the small gut and cecum caused this loop to fill with gas and come into view. It is probable that the anomaly caused a partial obstruction under certain favourable conditions.

It is interesting to note that since these cases were observed, Professor Whitnall (Anatomy) and Professor Simpsom (Histology and Embryology) have found two similar conditions in $\mathbf{5 0}$ bodies in the dissecting room of McGill University. In these latter there was no clinical history, and no evidence that the anomaly had contributed to the death.

The only similar cases that $I$ have found recorded in medical literature were reported in May, 1890, and published by W. H. Bennett, Surgeon to and Lecturer on Anatomy at St. George's Hospital, and Rolleston, ${ }^{1}$ Curator of the Museum. They reported three examples of the anomaly, their attention being called to the condition by the fact that it

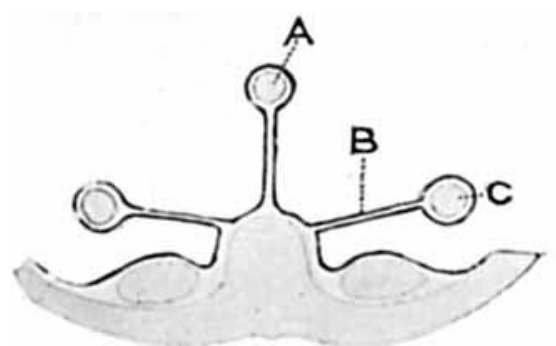

FIc. 283.-Character of the mesocolon in t1/e fotus. (A) Small intestine; (B) Mesocolon; (C) Ascendin: colon.

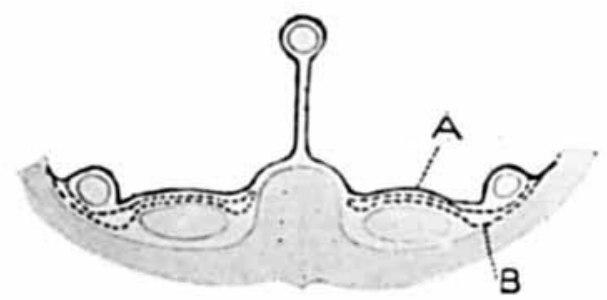

FIf. 281. - Normal arrangement of peritoneum; showing changes which occur in the fortal mesocolon. (A) Ascenting mesocolon fused to posterior abdominal wall; (B) D'eritoneal layers which are obliterated.

was "associated with, and probably instrumental in producing a fatal twisting of, the lower part of the ileum". The cases that were observed in the Royal Victoria Hospital differed from those reported by Bennett and Rolleston in the length and mobility of the cæcum and ascending colon. In their cases the cæcum had not descended into the right iliac fossa, but lay over the right kidney. Moreover, the cæcum was small and of the foetal type.

Normally, the ascending mesocolon of the fotus is flattened against the posterior abdominal wall on the right side, and fuses with the parietal peritoneum over the area shown in Fig. 282. Over this area the posterior layer of the fœetal mesocolon and the foetal parietal peritoneum are obliterated, and the anterior layer of the foetal mesocolon 


\section{DEFICIENCY OF MESENTERY OVER LOWER ILEUM 289}

becomes the parietal peritoneum of the adult. This is shown in Figs. 283, 284. This fusion of the ascending mesocolon normally begins at the level of the right colic flexure and extends downwards. In the cases described, this fusion has apparently extended low enough to include the mesentery of the terminal part of the ileum, as shown in Fig. 285.

A very possible cause of this extension of the area of fusion is the presence in the foctal stage of a 'genitomesenteric fold' (Fig. 286), which, by putting traction on the developing mesentery, may cause a fusion of the ileum to the posterior abdominal wall. Douglas G. Reid describes a 'genito-mesenteric fold' found in 11 of 20 fotuses examined. It passes from the inferior surface of the mesentery in the right half of the abdomen, forming an anteroposterior septum lying in the vertical plane, and incompletely dividing the portion of the abdominal cavity below the root of the mesentery into two compartments. The smaller of these is

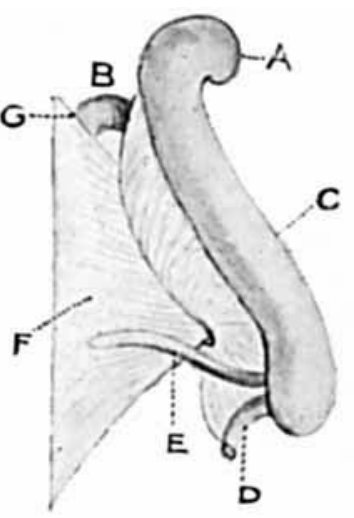

FIt: 286.-.Showing " genitomesenteric fold'. (A) Transverse colon; (B) Duodenum; (C) Ascending colon: (D) Ileum ; (E) Appendix: (F) Genitomesenteric fold ; (G) Duodenorenal ligament.

on the right side and contains the cacum, the larger on the left contains the pelvic colon.

The fold is triangular, and has two attached borders (superior and posterior) and one free (inferior). The posterior border is attached to the posterior abdominal wall along the line of the right spermatic or ovarian vessels. The superior, or mesenteric, border is attached to the inferior surface of the mesentery, often to a considerable extent. This attachment to the mesentery is generally exactly along the line of the iliac branch of the ileocolic artery. The upper part of the fold may persist as the free edge of the duodenorenal ligament of Huschke. Its lower part may persist in the adult, forming the medial boundary of the retrocolic fossa, passing from the ileum or appendix. It is the commonest cause of a retrocolic position of the appendix. "The fold may also assist in producing adhesions of the duodenum, cxcum, and ileum to the posterior abdominal wall. Adhesion in the adult, binding down the terminal part of the ileum, the crcum, the appendix, the mesentery, the meso-appendix, and even the 'bloodless' fold of Treves, need not be the result of pathological changes, unless the natural causes we have indicated for the adhesion can be excluded, although a genitomesenteric fold may not always be seen, even in the fotus" (p. 82).

\section{REFERENCES.}

1 Bennett and Roldeston, Jour. Anat. and Physiol., xxv.

2 Reid, Douglas G., "Studies of the Intestine and Peritoneum in the Human Fœtus," (6 figs.), Ibid., 1911 , xlv, 74 . 